Check for updates

Cite this: RSC Adv., 2017, 7, 52200

\title{
Peperomin E and its synthetic amino derivatives: potent agents targeting leukaemia stem cells $\uparrow$
}

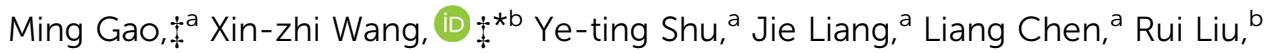 \\ Jing-yu Liang ${ }^{c}$ and Hong-mei Wen*a
}

Recent studies have described leukaemia stem cells (LSCS) as being central to the initiation, growth, and relapse of acute myelogenous leukaemia (AML). However, to date, very few small molecules have been shown to directly target this LSC population. The present study, for the first time, demonstrates that peperomin E (PepE), a naturally occurring secolignan, induces apoptosis in cultured AML and LSC-like cell lines, while sparing normal bone marrow stromal cells (hBMSCs). Furthermore, a series of eighteen amino derivatives was synthesised through a diastereoselective conjugate addition of several primary and secondary amines to the $\alpha$-methylene- $\boldsymbol{\gamma}$-butyrolactone moiety of PepE to increase its water-solubility and anti-AML/LSC activity. The synthesised amino analogues were evaluated for in vitro cytotoxicity against AML cell lines (KG-1, HL-60, THP-1) and an LSC-like cell line (KG-1a CD34 ${ }^{+}$CD38-). Most of the secondary open-chain amine derivatives showed enhanced water solubility and high activities against AML/LSC cells. The most potent compound, 6, derived from N-methylethanolamine, exhibited exclusive cytotoxicity against KG-1a CD34 ${ }^{+} \mathrm{CD}_{3} 8^{-}$cells, with an $\mathrm{IC}_{50}$ value of $0.5 \pm 0.1 \mu \mathrm{M}$, which was 13 -fold more potent than PepE. Moreover, compound 6 showed low toxicity against hBMSCs (inhibition of $11.2 \%$ at $50 \mu \mathrm{M})$. Preliminary mechanistic studies revealed that compound 6 could induce apoptosis of KG-1a $\mathrm{CD} 34^{+} \mathrm{CD} 38^{-}$cells. Therefore, we propose that compound 6 should be tested further for its in vivo activity and safety with the aim to develop it as a new type of drug for LSC-targeted therapy.

Received 6th September 2017 Accepted 4th November 2017

DOI: $10.1039 / c 7 r a 09928 b$

rsc.li/rsc-advances

\section{Introduction}

Acute myelogenous leukaemia (AML) is the most common leukaemia, characterised by the clonal expansion of immature myeloblasts initiating from rare leukemic stem cells (LSCs). ${ }^{1,2}$ As one of the most common types of lethal leukaemia, AML typically leads to death within weeks to months after clinical presentation. Current chemotherapy for AML commonly uses drugs, such as nucleoside analogues (e.g., cytarabine) and anthracyclines (e.g., idarubicin), which interfere with DNA replication and induce apoptosis primarily in replicating cells. ${ }^{3}$ Although remission can be achieved in most patients by standard chemotherapy, relapse is common and long-term survival is poor for most cases. ${ }^{2}$ It has been

\footnotetext{
${ }^{a}$ School of Pharmacy, Nanjing University of Chinese Medicines, Xianlin Avenue No. 138, Nanjing 210023, The People's Republic of China. E-mail: njwenhm@126.com; Fax: +86-025-858-11839; Tel: +86-025-858-11839

${ }^{b}$ Marine Drug Research and Development Center of Jiangsu Province, Nanjing University of Chinese Medicines, Xianlin Avenue No. 138, Nanjing 210023, The People's Republic of China. E-mail: wxzatnj@sina.com

'Department of Natural Medicinal Chemistry, China Pharmaceutical University, Tongjia Lane No. 24, 210009 Nanjing, The People's Republic of China

$\dagger$ Electronic supplementary information (ESI) available. See DOI: $10.1039 / \mathrm{c} 7 \mathrm{ra09928b}$

\$ These authors contributed equally to this work.
}

reported that LSCs are refractory to standard chemotherapy and thereby contribute to relapse. However, there are few compounds that can selectively inhibit LSCs. ${ }^{4}$ Therefore, novel therapeutic agents with selective activity against LSCs are urgently needed.

Peperomin E (PepE) is a naturally occurring secolignan, isolated from Peperomia dindygulensis (Piperaceae), a commonly used folk medicine to treat cancers in southern China. ${ }^{5}$ Although PepE was first to be extracted from nature plants, it can also be fully synthesized from phosphorylated cyclopropanes, which broadens its further study. ${ }^{6}$ There has been increasing interest in this compound, due to its remarkable activity against multiple cancer cells in vitro and in vivo with low toxicity against normal cells. ${ }^{7,8}$ The presence of the $\alpha$-methylene$\gamma$-butyrolactone moiety in this compound was reported to be essential for its antitumour properties. ${ }^{7}$ The exo-methylene moiety is considered important because of its reactivity toward biological nucleophiles, especially the thiol groups of cysteines in target proteins, which act as Michael acceptors. ${ }^{9}$ Recently, our laboratory discovered that PepE could exceptionally target and initiate apoptosis in AML cells (e.g., KG-1 cells) and AML stem cells (e.g., KG-1a CD $34^{+} \mathrm{CD} 38^{-}$cells), with high survival rates for normal bone marrow stromal cells (hBMSCs). Studies to elucidate the anti-AML mechanism of this promising agent are underway in our laboratory; however, preliminary 


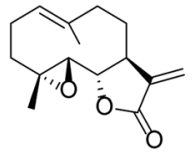

A

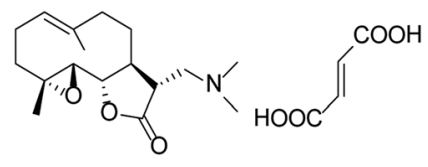

B
Fig. 1 Structures of PTL and its Michael adducts DMAPT.

experiments have suggested that NF-KB and DNA methyltransferases are the main targets of this compound (unpublished results).

Although PepE has shown potent activity against AML, its naked $\alpha$-methylene- $\gamma$-butyrolactone substructure is unstable in both acidic and basic conditions and imparts poor water solubility and bioavailability $;^{10}$ these issues have limited its potential therapeutic use in humans. Converting the $\alpha$-methylene- $\gamma$ butyrolactone moiety to its prodrug with a masking group, such as a primary or secondary amine, effectively increases its stability, water solubility, and bioavailability, while maintaining or even enhancing its biological activity. ${ }^{11-15}$ Amino adducts of $\alpha$-methylene- $\gamma$-butyrolactone have been previously prepared from sesquiterpene lactones, including helenalin, ambrosin, costunolide, alantolactone, and parthenolide (PTL). Among them, the dimethylamino analogue of PTL (DMAPT, Fig. 1) has advanced to a phase I clinical trial for evaluation as a treatment for AML. ${ }^{11,16-20}$

Therefore, the aim of the present study was to overcome the poor water solubility of PepE, while at least maintaining its activity, by derivatizing it with several primary and secondary amino compounds. These analogues were then converted into water-soluble organic/inorganic salts and assessed for their cytotoxicity against AML cells and LSCs. To the best of our knowledge, there is no published record available concerning the anti-AML or anti-LSC effects of PepE and its amino analogues.

\section{Experimental}

\section{Chemistry}

General methods. All commercial reagents and solvents were used without purification. All reactions were performed under a nitrogen atmosphere with dry solvents under anhydrous conditions. ${ }^{1} \mathrm{H}$ and ${ }^{13} \mathrm{C}$ NMR (1D and 2D) spectra were recorded with a $500 \mathrm{MHz}\left({ }^{1} \mathrm{H}\right.$ NMR: $500 \mathrm{MHz},{ }^{13} \mathrm{C}$ NMR: $125 \mathrm{MHz}$ ) Bruker spectrometer (Bruker, Germany) with tetramethylsilane (TMS) as the internal standard. Chemical shifts are reported in ppm, multiplicities are indicated by s (singlet), $\mathrm{d}$ (doublet), $\mathrm{t}$ (triplet), $\mathrm{q}$ (quartet), and $\mathrm{m}$ (multiplet). NMR data are reported as follows: chemical shift, multiplicity, coupling constants, and integration. Reactions were monitored by thin-layer chromatography (TLC) on GF-254 silica gel plates (Tsingdao Silica Gel Corp., Tsingdao, China). Visualisation was achieved using UV light $(254 \mathrm{~nm})$. A silica gel column was used for flash column chromatography (300-400 mesh, Tsingdao Silica Gel Corp., Tsingdao, China). High-resolution electrospray ionisation mass spectrometry (HRMS-ESI) was measured using a 5600 time-of- flight (TOF) system equipped with a TurboIonSpray ${ }^{\mathrm{TM}}$ source (Applied Biosystems, Foster City, USA) tested in positive ionisation mode. The electron-capture dissociation (ECD) spectra of each compound were recorded in methanol solution by the Jasco J-810 detector (Jasco Corp., USA). Infrared (IR) spectra were recorded on a ThermoNicolet IR-100 spectrometer with $\mathrm{NaCl}$ plates as a thin film dispersed from $\mathrm{CH}_{2} \mathrm{Cl}_{2}$ and are reported in wavenumbers $\left(\mathrm{cm}^{-1}\right)$.

General synthesis procedure for compounds 2-19. To a stirred solution of PepE $(0.2 \mathrm{mmol})$ and triethylamine $(0.6 \mathrm{mmol})$ in methanol $(4 \mathrm{~mL})$ the indicated amino compound $(0.2 \mathrm{mmol})$ was added. The reaction mixture was stirred at ambient temperature for $18 \mathrm{~h}$. After completion of the reaction (monitored by TLC), the reaction mixture was concentrated under reduced pressure to afford the crude compound. Water $(10 \mathrm{~mL})$ was added to the crude compound, and the solution was extracted with dichloromethane $(2 \times 10 \mathrm{~mL})$. The organic layer was dried over $\mathrm{Na}_{2} \mathrm{SO}_{4}$, concentrated, and then purified by flash column chromatography [hexane : EtOAc $=3: 1$ to $1: 1$ ] to yield the desired compounds 2-19. Purity of the compounds was established by HPLC analysis, confirming a purity $\geq 95 \%$. The characterisation data for these compounds are listed below.

(2S,3S)-6-Dimethylamino-2,6-dihydropeperomin $E$ (2). Pale yellow oil (yield 85\%); 98.5\% HPLC purity; IR (thin film) $\nu_{\max }$ 2904.94, 1758.52, 1633.46, 1508.44, 1450.62, 1431.89, 1130.91, 1091.96, 1042.72, 928.07, and $823.67 \mathrm{~cm}^{-1} ;{ }^{1} \mathrm{H}$ NMR $(500 \mathrm{MHz}$, $\left.\mathrm{CDCl}_{3}\right) \delta_{\mathrm{H}} 6.56(\mathrm{~d}, J=2.0 \mathrm{~Hz}, 1 \mathrm{H}), 6.51(\mathrm{~d}, J=1.5 \mathrm{~Hz}, 1 \mathrm{H}), 6.47$ (d, $J=2.0 \mathrm{~Hz}, 1 \mathrm{H}), 6.43(\mathrm{~d}, J=1.5 \mathrm{~Hz}, 1 \mathrm{H}), 5.93-5.96(\mathrm{~m}, 4 \mathrm{H})$, $4.36(\mathrm{dd}, J=9.0,12.5 \mathrm{~Hz}, 1 \mathrm{H}), 4.01(\mathrm{dd}, J=4.5,12.5 \mathrm{~Hz}, 1 \mathrm{H})$, $3.93(\mathrm{~s}, 3 \mathrm{H}), 3.91(3 \mathrm{H}, \mathrm{s}), 3.62(\mathrm{~d}, J=15.5 \mathrm{~Hz}, 1 \mathrm{H}), 3.36-3.43(\mathrm{~m}$, $1 \mathrm{H}), 2.53(\mathrm{dd}, J=9.5,15.5 \mathrm{~Hz}, 1 \mathrm{H}), 2.42-2.46(\mathrm{~m}, 1 \mathrm{H}), 2.20(\mathrm{dd}, J$ $=6.5,15.5 \mathrm{~Hz}, 1 \mathrm{H})$ and $2.10(\mathrm{~s}, 6 \mathrm{H}) \mathrm{ppm} ;{ }^{13} \mathrm{C} \mathrm{NMR}(125 \mathrm{MHz}$, $\left.\mathrm{CDCl}_{3}\right) \delta_{\mathrm{C}} 178.7,149.5,149.2,143.6,143.5,136.6,136.9,134.2$, 134.1, 108.1, 108.0, 101.9, $101.5(\times 2), 101.3,70.6,58.1,57.0$, $56.8,55.4,45.8(\times 2), 44.7$ and $41.4 \mathrm{ppm}$; HRMS-ESI calcd for $\mathrm{C}_{24} \mathrm{H}_{28} \mathrm{NO}_{8}[\mathrm{M}+\mathrm{H}]^{+} 458.1815$, found 458.1797 .

(2S,3S)-6-Diethylamino-2,6-dihydropeperomin E (3). Pale yellow oil (yield 87\%); 97.0\% HPLC purity; IR (thin film) $\nu_{\max } 2919.07$, 1763.85, 1633.47, 1508.71, 1450.46, 1432.51, 1132.31, 1091.86, $1042.90,926.56$, and $818.35 \mathrm{~cm}^{-1} ;{ }^{1} \mathrm{H}$ NMR $\left(500 \mathrm{MHz}, \mathrm{CDCl}_{3}\right) \delta_{\mathrm{H}}$ $6.54(\mathrm{~d}, J=1.5 \mathrm{~Hz}, 1 \mathrm{H}), 6.48(\mathrm{~d}, J=2.0 \mathrm{~Hz}, 1 \mathrm{H}), 6.42(\mathrm{~d}, J=$ $1.5 \mathrm{~Hz}, 2 \mathrm{H}), 5.93-5.96(\mathrm{~m}, 4 \mathrm{H}), 4.36(\mathrm{dd}, J=8.5,12.0 \mathrm{~Hz}, 1 \mathrm{H})$, $4.05(\mathrm{dd}, J=3.5,12.0 \mathrm{~Hz}, 1 \mathrm{H}), 3.92(\mathrm{~s}, 3 \mathrm{H}), 3.91(\mathrm{~s}, 3 \mathrm{H}), 3.65(\mathrm{~d}, J$ $=15.5 \mathrm{~Hz}, 1 \mathrm{H}), 3.27-3.31(\mathrm{~m}, 1 \mathrm{H}), 2.69(\mathrm{dd}, J=12.0,18.0 \mathrm{~Hz}$, $1 \mathrm{H}), 2.42-2.54(\mathrm{~m}, 4 \mathrm{H}), 2.39(\mathrm{dd}, J=9.0,16.5 \mathrm{~Hz}, 1 \mathrm{H})$, and 0.98 (t, $J=9.5 \mathrm{~Hz}, 6 \mathrm{H}) \mathrm{ppm} ;{ }^{13} \mathrm{C} \mathrm{NMR}\left(125 \mathrm{MHz}, \mathrm{CDCl}_{3}\right) \delta_{\mathrm{C}} 178.9$, $149.5,149.2$, 143.6, 143.5, 137.1, 136.6, 134.2, 134.1, 108.3, 108.2, 101.8, 101.5, 101.4, 101.3, 70.6, 60.3, 56.9, 56.8, 56.7, 55.1, 44.7, 41.9, 41.8, 20.3 and $11.8 \mathrm{ppm}$; HRMS-ESI calcd for $\mathrm{C}_{26} \mathrm{H}_{32} \mathrm{NO}_{8}[\mathrm{M}+\mathrm{H}]^{+}$486.2128, found 486.2110.

(2S,3S)-6-Ethyl(methyl)amino-2,6-dihydropeperomin E (4). Pale yellow oil (yield 81\%); 100.0\% HPLC purity; IR (thin film) $\nu_{\max }$ 2920.97, 1767.51, 1633.26, 1508.64, 1450.71, 1432.08, 1131.95, 1091.38, 1042.65, 927.23, and $827.68 \mathrm{~cm}^{-1} ;{ }^{1} \mathrm{H}$ NMR $(500 \mathrm{MHz}$, $\left.\mathrm{CDCl}_{3}\right) \delta_{\mathrm{H}} 6.56(\mathrm{~d}, J=2.0 \mathrm{~Hz}, 1 \mathrm{H}), 6.50(\mathrm{~d}, J=2.0 \mathrm{~Hz}, 1 \mathrm{H}), 6.44$ (d, $J=1.5 \mathrm{~Hz}, 2 \mathrm{H}), 6.42(\mathrm{~d}, J=2.0 \mathrm{~Hz}, 2 \mathrm{H}), 5.93-5.97(\mathrm{~m}, 4 \mathrm{H})$, $4.37(\mathrm{dd}, J=9.0,12.0 \mathrm{~Hz}, 1 \mathrm{H}), 4.04(\mathrm{dd}, J=3.0,12.0 \mathrm{~Hz}, 1 \mathrm{H})$, 
3.92 (s, 3H), 3.91 (s, 3H), $3.63(\mathrm{~d}, J=15.5 \mathrm{~Hz}, 1 \mathrm{H}), 3.30-3.37(\mathrm{~m}$, $1 \mathrm{H}), 2.62$ (dd, $J=10.5,16.0 \mathrm{~Hz}, 1 \mathrm{H}), 2.44-2.50$ (m, 2H), 2.31$2.36(\mathrm{~m}, 2 \mathrm{H}), 2.05(\mathrm{~s}, 3 \mathrm{H})$, and $1.03(\mathrm{t}, J=8.0 \mathrm{~Hz}, 3 \mathrm{H}) \mathrm{ppm} ;{ }^{13} \mathrm{C}$ NMR $\left(125 \mathrm{MHz}, \mathrm{CDCl}_{3}\right) \delta_{\mathrm{C}} 179.0,149.4,149.2,143.6,143.5$, $137.1,136.6,134.2$, 134.1, 108.4, 108.3, 101.7, 101.5, 101.4, 101.2, 70.6, 57.0, 56.9, 56.8, 54.7, 52.7, 44.9, 42.2, and 11.3 $(\times 2)$ ppm; HRMS-ESI calcd for $\mathrm{C}_{25} \mathrm{H}_{30} \mathrm{NO}_{8}[\mathrm{M}+\mathrm{H}]^{+}$472.1971, found 472.1949 .

(2S,3S)-6-Methyl(propyl)amino-2,6-dihydropeperomin $\quad E \quad$ (5). Pale yellow oil (yield 78\%); 99.0\% HPLC purity; IR (thin film) $\nu_{\max } 2960.34,1770.08,1633.32,1508.85,1451.98,1432.89$, 1132.41, 1092.48, 1044.01, 929.64, and 828.77 $\mathrm{cm}^{-1} ;{ }^{1} \mathrm{H}$ NMR $\left(500 \mathrm{MHz}, \mathrm{CDCl}_{3}\right) \delta_{\mathrm{H}} 6.55(\mathrm{~d}, J=2.0 \mathrm{~Hz}, 1 \mathrm{H}), 6.48(\mathrm{~d}, J=2.0 \mathrm{~Hz}$, $1 \mathrm{H}), 6.44(\mathrm{~d}, J=2.0 \mathrm{~Hz}, 1 \mathrm{H}), 6.42(\mathrm{~d}, J=2.0 \mathrm{~Hz}, 1 \mathrm{H}), 5.93-5.96$ $(\mathrm{m}, 4 \mathrm{H}), 4.36(\mathrm{dd}, J=9.0,12.0 \mathrm{~Hz}, 1 \mathrm{H}), 4.02(\mathrm{dd}, J=4.0,12.0 \mathrm{~Hz}$, $1 \mathrm{H}), 3.92(\mathrm{~s}, 3 \mathrm{H}), 3.91(\mathrm{~s}, 3 \mathrm{H}), 3.62$ (d, $J=15.5 \mathrm{~Hz}, 1 \mathrm{H}), 3.36-3.39$ $(\mathrm{m}, 1 \mathrm{H}), 2.62(\mathrm{dd}, J=10.0,15.5 \mathrm{~Hz}, 1 \mathrm{H}), 2.46-2.49(\mathrm{~m}, 1 \mathrm{H}), 2.29$ (dd, $J=6.5,16.0 \mathrm{~Hz}, 1 \mathrm{H}), 2.24(\mathrm{t}, J=9.5 \mathrm{~Hz}, 2 \mathrm{H}), 2.03(\mathrm{~s}, 3 \mathrm{H})$, 1.43-1.50 (m, 2H), $0.92(\mathrm{t}, 3 \mathrm{H}, J=9.0 \mathrm{~Hz}) ;{ }^{13} \mathrm{C}$ NMR $(125 \mathrm{MHz}$, $\left.\mathrm{CDCl}_{3}\right) \delta_{\mathrm{C}} 178.9,149.5,149.2,143.6,143.5,137.1,136.6,134.2$, 134.1, 108.3, 108.2, 101.8, 101.5, 101.4, 101.3, 70.6, 60.3, 56.9, 56.8, 56.7, 55.1, 44.7, 41.8, 41.7, 20.3, 11.8 ppm; HRMS-ESI calcd for $\mathrm{C}_{26} \mathrm{H}_{32} \mathrm{NO}_{8}[\mathrm{M}+\mathrm{H}]^{+} 486.2128$, found 486.2102 .

(2S,3S)-6-Methyl(hydroxyethyl)amino-2,6-dihydropeperomin $E$ (6). Colourless powder (yield 82\%); 100.0\% HPLC purity; IR (thin film) $\nu_{\max } 3445.62,2917.98,1767.13,1633.65,1507.61$, 1451.75, 1435.25, 1192.73, 1135.07, 1090.75, 1042.05, 927.53, and $852.33 \mathrm{~cm}^{-1} ;{ }^{1} \mathrm{H}$ NMR $\left(500 \mathrm{MHz}, \mathrm{CDCl}_{3}\right) \delta_{\mathrm{H}} 6.50(\mathrm{~d}, J=$ $2.0 \mathrm{~Hz}, 1 \mathrm{H}), 6.48(\mathrm{~d}, J=2.0 \mathrm{~Hz}, 1 \mathrm{H}), 6.44(\mathrm{~d}, J=2.0 \mathrm{~Hz}, 1 \mathrm{H}), 6.41$ $(\mathrm{d}, J=2.0 \mathrm{~Hz}, 1 \mathrm{H}), 5.95-5.97(\mathrm{~m}, 4 \mathrm{H}), 4.37(\mathrm{dd}, J=9.0,12.0 \mathrm{~Hz}$, $1 \mathrm{H}), 4.00(\mathrm{dd}, J=5.5,12.0 \mathrm{~Hz}, 1 \mathrm{H}), 3.92(\mathrm{~s}, 6 \mathrm{H}), 3.64$ (d, $J=$ $15.5 \mathrm{~Hz}, 1 \mathrm{H}), 3.62-3.58(\mathrm{~m}, 2 \mathrm{H}), 3.14-3.20$ (m, 1H), 2.54-2.64 (m, 3H), 2.42-2.53 (m, 2H), $2.08(\mathrm{~s}, 3 \mathrm{H}) ;{ }^{13} \mathrm{C}$ NMR $(125 \mathrm{MHz}$, $\left.\mathrm{CDCl}_{3}\right) \delta_{\mathrm{C}} 178.5,149.5,149.3,143.7,143.6,136.6,136.4,134.3$ $(\times 2), 108.2,108.1,101.6,101.5,101.2(\times 2), 70.5,59.5,58.9,57.4$, $56.9(\times 2), 55.4,44.4,42.4$, and 41.7 ppm; HRMS-ESI calcd for $\mathrm{C}_{25} \mathrm{H}_{30} \mathrm{NO}_{9}[\mathrm{M}+\mathrm{H}]^{+}$488.1921, found 488.1890.

(2S,3S)-6-Methylglycine-2,6-dihydropeperomin $E$ (7). Pale yellow oil (yield 64\%); 98.5\% HPLC purity; IR (thin film) $\nu_{\max }$ 3420.91, 2917.98, 1767.13, 1633.65, 1507.61, 1451.75, 1435.25, 1192.73, 1135.07, 1090.75, 1042.05, and $928.05 \mathrm{~cm}^{-1} ;{ }^{1} \mathrm{H}$ NMR $\left(500 \mathrm{MHz}, \mathrm{CDCl}_{3}\right) \delta_{\mathrm{H}} 6.48(\mathrm{~d}, J=2.0 \mathrm{~Hz}, 1 \mathrm{H}), 6.47(\mathrm{~d}, J=2.0 \mathrm{~Hz}$, $1 \mathrm{H}), 6.40$ (d, $J=1.5 \mathrm{~Hz}, 1 \mathrm{H}), 6.28$ (d, $J=1.5 \mathrm{~Hz}, 1 \mathrm{H}), 5.96-5.97$ $(\mathrm{m}, 4 \mathrm{H}), 4.35(\mathrm{dd}, J=9.0,12.0 \mathrm{~Hz}, 1 \mathrm{H}), 4.01(\mathrm{dd}, J=5.5,12.0 \mathrm{~Hz}$, $1 \mathrm{H}), 3.92(\mathrm{~s}, 3 \mathrm{H}), 3.91$ (s, 3H), 3.68 (d, $J=14.5 \mathrm{~Hz}, 1 \mathrm{H}), 3.50-3.59$ (m, $1 \mathrm{H}), 3.09$ (q, $J=9.5 \mathrm{~Hz}, 2 \mathrm{H}), 2.51(\mathrm{dd}, J=9.5,14.5 \mathrm{~Hz}, 1 \mathrm{H})$, 2.18-2.40 (m, 2H), 2.06 (s, 3H) ppm; ${ }^{13} \mathrm{C} \mathrm{NMR} \mathrm{(125} \mathrm{MHz,} \mathrm{CDCl}_{3}$ ) $\delta_{\mathrm{C}} 174.0,170.7,149.6,149.3,143.7,143.4,136.1(\times 2), 135.9$, 108.5, 108.1, 101.6, 101.5 (×2), 101.2, 69.7, 60.7, 60.4, 57.0, 56.9, 55.3, 45.8, $45.6(\times 2)$, and $42.5 \mathrm{ppm}$; HRMS-ESI calcd for $\mathrm{C}_{25} \mathrm{H}_{28} \mathrm{NO}_{10}[\mathrm{M}+\mathrm{H}]^{+}$502.1718, found 502.1720.

(2S,3S)-6-Pyrrolidin-2,6-dihydropeperomin E (8). Pale yellow oil (yield 89\%); 99.5\% HPLC purity; IR (thin film) $\nu_{\max } 2926.35$, 2789.59, 1632.74, 1609.01, 1507.80, 1449.91, 1431.09, 1128.95, 1089.54, 1043.00, 927.88, and $828.35 \mathrm{~cm}^{-1} ;{ }^{1} \mathrm{H}$ NMR $(500 \mathrm{MHz}$, $\left.\mathrm{CDCl}_{3}\right) \delta_{\mathrm{H}} 6.59(\mathrm{~d}, J=2.0 \mathrm{~Hz}, 1 \mathrm{H}), 6.57(\mathrm{~d}, J=1.5 \mathrm{~Hz}, 1 \mathrm{H}), 6.54$ $(\mathrm{d}, J=1.5 \mathrm{~Hz}, 1 \mathrm{H}), 6.45$ (d, $J=1.5 \mathrm{~Hz}, 1 \mathrm{H}), 5.89-5.95(\mathrm{~m}, 4 \mathrm{H})$,
4.01 (dd, $J=3.5,12 \mathrm{~Hz}, 1 \mathrm{H}), 3.90$ (s, 6H), 3.89 (d, $J=15.5 \mathrm{~Hz}$, $1 \mathrm{H}), 3.52$ (dd, $J=4.5,15.5 \mathrm{~Hz}, 1 \mathrm{H}), 3.41-3.48(\mathrm{~m}, 1 \mathrm{H}), 2.47-2.56$ (m, 3H), 2.39-2.46 (m, 3H), 2.05-2.13 (m, 1H), 1.94-1.98 (m, $2 \mathrm{H})$, and 1.88-1.92 (m, 2H) ppm; ${ }^{13} \mathrm{C} \mathrm{NMR}\left(125 \mathrm{MHz}, \mathrm{CDCl}_{3}\right) \delta_{\mathrm{C}}$ 173.1 , 149.2, 148.9, 143.7, 143.4, 138.5, 138.2, 133.7, 133.6, $108.3,107.7,101.7,101.4,101.2,101.1,71.2,58.2$, 57.0, 56.7 $(\times 2), 54.3(\times 2), 44.3,43.7,23.6(\times 2)$ ppm; HRMS-ESI calcd for $\mathrm{C}_{26} \mathrm{H}_{30} \mathrm{NO}_{8}[\mathrm{M}+\mathrm{H}]^{+}$484.1971, found 484.1964.

(2S,3S)-6-Morpholin-2,6-dihydropeperomin E (9). Pale yellow oil (yield 82\%); 98.0\% HPLC purity; IR (thin film) $\nu_{\max } 2921.57$, 1759.13, 1636.71, 1508.44, 1449.62, 1436.61, 1118.32, 1092.17, 1045.22, and $925.09 \mathrm{~cm}^{-1} ;{ }^{1} \mathrm{H}$ NMR $\left(500 \mathrm{MHz}, \mathrm{CDCl}_{3}\right) \delta_{\mathrm{H}} 6.56(\mathrm{~d}$, $J=2.0 \mathrm{~Hz}, 1 \mathrm{H}), 6.50(\mathrm{~d}, J=2.0 \mathrm{~Hz}, 1 \mathrm{H}), 6.41(\mathrm{~d}, J=2.0 \mathrm{~Hz}, 2 \mathrm{H})$, 5.92-5.97 (m, 4H), 4.36 (dd, $J=9.0,12.0 \mathrm{~Hz}, 1 \mathrm{H}), 4.03(\mathrm{~d}, J=4.5$, $12.0 \mathrm{~Hz}, 1 \mathrm{H}), 3.93(\mathrm{~s}, 3 \mathrm{H}), 3.91(\mathrm{~s}, 3 \mathrm{H}), 3.63-3.69(\mathrm{~m}, 4 \mathrm{H}), 3.61(\mathrm{~d}$, $J=15.0 \mathrm{~Hz}, 1 \mathrm{H}), 3.33-3.40(\mathrm{~m}, 1 \mathrm{H}), 2.59$ (dd, $J=10.5,15.5 \mathrm{~Hz}$, $1 \mathrm{H}), 2.51-2.62(\mathrm{~m}, 1 \mathrm{H}), 2.32-2.51(\mathrm{~m}, 3 \mathrm{H})$, and $2.20-2.26(\mathrm{~m}$, $2 \mathrm{H}) ;{ }^{13} \mathrm{C}$ NMR $\left(125 \mathrm{MHz}, \mathrm{CDCl}_{3}\right) \delta_{\mathrm{C}} 178.5,149.5,149.2,143.6$, 143.4, 136.8, 136.6, 134.2, 134.1, 108.7, 108.3, 101.5, $101.4(\times 2)$, $101.1,70.6,67.0,66.8,57.2,57.0,56.9,55.0,52.0,43.9$, and 41.8 ppm; HRMS-ESI calcd for $\mathrm{C}_{26} \mathrm{H}_{30} \mathrm{NO}_{9}[\mathrm{M}+\mathrm{H}]^{+}$500.1921, found 500.1895 .

(2S,3S)-6-Piperidin-2, 6-dihydropeperomin E (10). Pale yellow oil (yield 85\%); 99.5\% HPLC purity; IR (thin film) $\nu_{\max } 2922.04$, 2851, 1767.84, 1633.33, 1508.21, 1450.80, 1431.92, 1132.96, 1090.35, 1043.20, 927.59, and $851.45 \mathrm{~cm}^{-1} ;{ }^{1} \mathrm{H}$ NMR $(500 \mathrm{MHz}$, $\left.\mathrm{CDCl}_{3}\right) \delta_{\mathrm{H}} 6.58(\mathrm{~d}, J=2.0 \mathrm{~Hz}, 1 \mathrm{H}), 6.51(\mathrm{~d}, J=2.0 \mathrm{~Hz}, 1 \mathrm{H}), 6.42$ $(\mathrm{d}, J=2.5 \mathrm{~Hz}, 2 \mathrm{H}), 5.93-5.99(\mathrm{~m}, 4 \mathrm{H}), 4.36(\mathrm{dd}, J=8.5,11.5 \mathrm{~Hz}$, $1 \mathrm{H}), 4.02(\mathrm{~d}, J=6.0,12.5 \mathrm{~Hz}, 1 \mathrm{H}), 3.93(\mathrm{~s}, 3 \mathrm{H}), 3.91(\mathrm{~s}, 3 \mathrm{H}), 3.61$ $(\mathrm{d}, J=15.5 \mathrm{~Hz}, 1 \mathrm{H}), 3.35-3.39(\mathrm{~m}, 1 \mathrm{H}), 3.52(\mathrm{dd}, J=4.5,15.5 \mathrm{~Hz}$, $1 \mathrm{H}), 2.56$ (dd, $J=10.5,15.5 \mathrm{~Hz}, 1 \mathrm{H}), 2.48-2.51$ (m, 1H), 2.22$2.47(\mathrm{~m}, 3 \mathrm{H}), 2.12-2.16(\mathrm{~m}, 1 \mathrm{H}), 1.52-1.57(\mathrm{~m}, 4 \mathrm{H})$, and $1.40-$ $1.45(\mathrm{~m}, 2 \mathrm{H}) \mathrm{ppm} ;{ }^{13} \mathrm{C} \mathrm{NMR}\left(125 \mathrm{MHz}, \mathrm{CDCl}_{3}\right) \delta_{\mathrm{C}} 179.1,149.4$, 149.2, 143.6, 143.4, 137.1, 136.7, 134.2, 134.1, 108.4, 108.2, 101.5, $101.4(\times 2), 101.2,70.7,57.5,57.0,56.8,55.0,54.8,44.2$, 41.8, $29.7(\times 2), 22.7$ ppm; HRMS-ESI calcd for $\mathrm{C}_{26} \mathrm{H}_{30} \mathrm{NO}_{9}[\mathrm{M}+$ $\mathrm{H}]^{+} 498.2128$, found 498.2119 .

(2S,3S)-6-(9-Methylpiperidin)-2,6-dihydropeperomin E (11). Pale yellow oil (yield 78\%); 97.0\% HPLC purity; IR (thin film) $\nu_{\max }$ 2922.87, 1763.10, 1633.91, 1509.18, 1450.56, 1433.50, 1131.33, 1090.99, 1043.45, 926.68, and $847.34 \mathrm{~cm}^{-1} ;{ }^{1} \mathrm{H}$ NMR $(500 \mathrm{MHz}$, $\left.\mathrm{CDCl}_{3}\right) \delta_{\mathrm{H}} 6.56(\mathrm{~d}, J=2.0 \mathrm{~Hz}, 1 \mathrm{H}), 6.51(\mathrm{~d}, J=1.5 \mathrm{~Hz}, 1 \mathrm{H}), 6.42$ $(\mathrm{d}, J=1.5 \mathrm{~Hz}, 1 \mathrm{H}), 6.41(\mathrm{~d}, J=2.0 \mathrm{~Hz}, 1 \mathrm{H}), 5.93-5.96(\mathrm{~m}, 4 \mathrm{H})$, 4.35 (dd, $J=8.5,12.0 \mathrm{~Hz}, 1 \mathrm{H}), 4.03$ (dd, $J=4.5,13.5 \mathrm{~Hz}, 1 \mathrm{H}$ ), $3.93(\mathrm{~s}, 3 \mathrm{H}), 3.91(\mathrm{~s}, 3 \mathrm{H}), 3.61(\mathrm{~d}, J=15.5 \mathrm{~Hz}, 1 \mathrm{H}), 3.39-3.41(\mathrm{~m}$, $1 \mathrm{H}), 2.57-2.66(\mathrm{~m}, 1 \mathrm{H}), 2.51$ (dd, $J=15.5,10.0 \mathrm{~Hz}, 1 \mathrm{H}), 2.46-$ $2.49(\mathrm{~m}, 1 \mathrm{H}), 2.30-2.34(\mathrm{~m}, 1 \mathrm{H}), 2.24(\mathrm{dd}, J=15.5,6.0 \mathrm{~Hz}, 1 \mathrm{H})$, 1.98-2.03 (m, 1H), 1.81-1.84 (m, 1H), 1.61-1.63 (m, 1H), 1.45$1.48(\mathrm{~m}, 1 \mathrm{H}), 1.19-1.25(\mathrm{~m}, 2 \mathrm{H})$, and $0.93(\mathrm{~d}, J=8.0 \mathrm{~Hz}$, $3 \mathrm{H}) \mathrm{ppm} ;{ }^{13} \mathrm{C}$ NMR $\left(125 \mathrm{MHz}, \mathrm{CDCl}_{3}\right) \delta_{\mathrm{C}} 179.1,149.4,149.2$, 143.6, 143.4, 137.1, 136.7, 134.1 (×2), 108.4, 108.3, 101.7, 101.6, 101.5, 101.4, 70.7, 60.4, 57.0, 56.9, 55.1, 54.9, 53.6, 44.4, 41.7, 34.1, 30.5, 21.7, 14.2 ppm; HRMS-ESI calcd for $\mathrm{C}_{28} \mathrm{H}_{34} \mathrm{NO}_{8}[\mathrm{M}+$ $\mathrm{H}]^{+}$512.2284, found 512.2270.

(2S,3S)-6-(9-Hydroxymethylpiperidin)-2,6-dihydropeperomin $E$ (12). Pale yellow oil (yield 73\%); 96.4\% HPLC purity; IR (thin film) $\nu_{\max } 3430.79$, 2919.26, 1767.42, 1633.44, 1508.59, 1450.63, 
1432.37, 1315.59, 1134.16, 1090.66, 1042.21, 925.88, and $828.06 \mathrm{~cm}^{-1} ;{ }^{1} \mathrm{H}$ NMR (500 MHz, $\left.\mathrm{CDCl}_{3}\right) 6.56(\mathrm{~d}, J=2.0 \mathrm{~Hz}, 1 \mathrm{H})$, $6.50(\mathrm{~d}, J=2.0 \mathrm{~Hz}, 1 \mathrm{H}), 6.41(\mathrm{~d}, J=2.0 \mathrm{~Hz}, 2 \mathrm{H}), 5.92-5.96(\mathrm{~m}$, $4 \mathrm{H}), 4.35$ (dd, $J=8.5,12.0 \mathrm{~Hz}, 1 \mathrm{H}), 4.01$ (dd, $J=4.5,12.0 \mathrm{~Hz}$, $1 \mathrm{H}), 3.93(\mathrm{~s}, 3 \mathrm{H}), 3.91(\mathrm{~s}, 3 \mathrm{H}), 3.61$ (d, $J=15.5 \mathrm{~Hz}, 1 \mathrm{H}), 3.52(\mathrm{~d}, J$ $=8.0 \mathrm{~Hz}, 2 \mathrm{H}), 3.36-3.38(\mathrm{~m}, 1 \mathrm{H}), 2.61-2.72(\mathrm{~m}, 1 \mathrm{H}), 2.57(\mathrm{dd}, J=$ 15.6, $10.0 \mathrm{~Hz}, 1 \mathrm{H}), 2.41-2.45(\mathrm{~m}, 1 \mathrm{H}), 2.35-2.40(\mathrm{~m}, 1 \mathrm{H}), 2.24$ (dd, $J=15.6,5.8 \mathrm{~Hz}, 1 \mathrm{H}), 2.00-2.06(\mathrm{~m}, 1 \mathrm{H}), 1.74-1.80(\mathrm{~m}, 1 \mathrm{H})$, 1.72-1.74 (m, 2H), 1.59-1.63 (m, 2H), 1.63-1.66 (m, 2H), 1.44$1.47(\mathrm{~m}, 1 \mathrm{H}) ;{ }^{13} \mathrm{C} \mathrm{NMR}\left(125 \mathrm{MHz}, \mathrm{CDCl}_{3}\right) \delta_{\mathrm{C}} 179.0,149.5,149.2$, 143.6, 143.4, 137.0, 136.7, 134.2, 134.1, 108.5, 108.3, $101.5(\times 2)$, 101.4, 101.2, 70.7, 67.8, 57.1, 57.0, 56.9, 55.1, 54.5, 53.2, 44.4, 41.7, 38.3, 29.0, and $28.5 \mathrm{ppm}$; HRMS-ESI calcd for $\mathrm{C}_{28} \mathrm{H}_{34} \mathrm{NO}_{9}$ $[\mathrm{M}+\mathrm{H}]^{+}$528.2234, found 528.2213.

(2S,3S)-6-Ethylamino-2,6-dihydropeperomin E (13). Pale yellow oil (yield 65\%); 98.0\% HPLC purity; IR (thin film) 3444.34, 2910.17, 1765.11, 1631.23, 1501.60, 1451.70, 1431.11, 1120.21, 1031.18, 1040.15, 920.20, and $825.12 \mathrm{~cm}^{-1}$; ${ }^{1} \mathrm{H}$ NMR $(500 \mathrm{MHz}$, $\left.\mathrm{CDCl}_{3}\right) \delta_{\mathrm{H}} 6.55(\mathrm{~d}, J=2.0 \mathrm{~Hz}, 1 \mathrm{H}), 6.49(\mathrm{~d}, J=1.5 \mathrm{~Hz}, 1 \mathrm{H}), 6.44$ $(\mathrm{d}, J=1.5 \mathrm{~Hz}, 1 \mathrm{H}), 6.42(\mathrm{~d}, J=2.0 \mathrm{~Hz}, 1 \mathrm{H}), 5.92-5.96(\mathrm{~m}, 4 \mathrm{H})$, 4.36 (dd, $J=8.5,12.0 \mathrm{~Hz}, 1 \mathrm{H}), 4.01$ (dd, $J=4.5,12.0 \mathrm{~Hz}, 1 \mathrm{H}$ ), $3.91(\mathrm{~s}, 3 \mathrm{H}), 3.89$ (s, 3H), $3.62(\mathrm{~d}, J=15.0 \mathrm{~Hz}, 1 \mathrm{H}), 3.33-3.37(\mathrm{~m}$, $1 \mathrm{H}), 2.61$ (dd, $J=10.0,15.5 \mathrm{~Hz}, 1 \mathrm{H}), 2.43-2.53(\mathrm{~m}, 2 \mathrm{H}), 1.02(\mathrm{t}, J$ $=8.5 \mathrm{~Hz}, 3 \mathrm{H}) ;{ }^{13} \mathrm{C} \mathrm{NMR}\left(125 \mathrm{MHz}, \mathrm{CDCl}_{3}\right) \delta_{\mathrm{C}} 179.0,149.4,149.2$, 143.6, 143.4, 137.0, 136.6, 134.2, 134.1, 108.1 (×2), 101.8, 101.6, 101.5, 101.4, 70.6, 56.9, 56.4, 56.8, 55.0, 44.7, 42.1, 41.5, and 12.1 ppm; HRMS-ESI calcd for $\mathrm{C}_{24} \mathrm{H}_{28} \mathrm{NO}_{8}[\mathrm{M}+\mathrm{H}]^{+} 458.1815$, found 458.1820 .

(2S,3S)-6-(4"'-Chloropyridinemethylamino)-2, 6-dihydropeperomin $E$ (14). Pale yellow oil (yield 79\%); $100.0 \%$ HPLC purity; IR (thin film) $\nu_{\max } 3441.55,2902.33,1765.23,1633.21,1507.99,1452.10$, 1432.43, 1315.90, 1042.06, 927.21, and $826.15 \mathrm{~cm}^{-1} ;{ }^{1} \mathrm{H}$ NMR $(500$ $\left.\mathrm{MHz}, \mathrm{CDCl}_{3}\right) 8.26(\mathrm{~d}, J=2.7 \mathrm{~Hz}, 1 \mathrm{H}), 7.59(\mathrm{dd}, J=2.7,10.2 \mathrm{~Hz}$, $1 \mathrm{H}), 7.31(\mathrm{~d}, J=10.2 \mathrm{~Hz}, 1 \mathrm{H}), 6.48(\mathrm{~d}, J=2.0 \mathrm{~Hz}, 1 \mathrm{H}), 6.42(\mathrm{~d}, J=$ $2.0 \mathrm{~Hz}, 1 \mathrm{H}), 6.40$ (d, $J=2.0 \mathrm{~Hz}, 2 \mathrm{H}), 5.92-5.95(\mathrm{~m}, 4 \mathrm{H}), 4.35$ (dd, $J$ $=9.0,12.0 \mathrm{~Hz}, 1 \mathrm{H}), 3.92(\mathrm{~s}, 3 \mathrm{H}), 3.89(\mathrm{~s}, 3 \mathrm{H}), 3.87(\mathrm{dd}, J=4.0$, $12.0 \mathrm{~Hz}, 1 \mathrm{H}), 3.59-3.64(\mathrm{~m}, 3 \mathrm{H}), 3.23-3.28(\mathrm{~m}, 1 \mathrm{H}), 2.67$ (dd, $J=$ $5.5,15.2 \mathrm{~Hz}, 1 \mathrm{H}), 2.52-2.55(\mathrm{~m}, 1 \mathrm{H})$, and $2.30(\mathrm{dd}, J=7.5,15.2 \mathrm{~Hz}$, $1 \mathrm{H}) \mathrm{ppm} ;{ }^{13} \mathrm{C}$ NMR $\left(125 \mathrm{MHz}, \mathrm{CDCl}_{3}\right) \delta_{\mathrm{C}} 175.5,149.1,149.0$, 143.6, 143.5, 139.5, 138.9, 138.3, 133.9, $133.8(\times 2), 128.7,128.6$, 128.0, 127.8, 107.8, 107.7, 101.6, 101.4, 101.3, 101.2, 70.9, 56.9, 56.8, 53.7, 51.6, 45.8, and $42.2 \mathrm{ppm}$; HRMS-ESI calcd for $\mathrm{C}_{28} \mathrm{H}_{28} \mathrm{ClN}_{2} \mathrm{O}_{8}[\mathrm{M}+\mathrm{H}]^{+}$555.1534, found 555.1508.

(2S,3S)-6-Benzylamino-2,6-dihydropeperomin $E$ (15). Pale yellow oil (yield 87\%); 96.4\% HPLC purity; IR (thin film) $\nu_{\max }$ 3404.80, 2922.65, 1762.13, 1633.55, 1508.00, 1451.40, 1431.05, $1313.60,1194.09,1130.23,1089.83,1043.31,928.59$, and $828.26 \mathrm{~cm}^{-1}$; ${ }^{1} \mathrm{H}$ NMR (500 MHz, $\left.\mathrm{CDCl}_{3}\right) 7.31-7.37(\mathrm{~m}, 4 \mathrm{H})$, $7.20-7.27(\mathrm{~m}, 1 \mathrm{H}), 6.53(\mathrm{~d}, J=2.0 \mathrm{~Hz}, 1 \mathrm{H}), 6.49(\mathrm{~d}, J=2.0 \mathrm{~Hz}$, $1 \mathrm{H}), 6.44(\mathrm{~d}, J=2.0 \mathrm{~Hz}, 1 \mathrm{H}), 6.40(\mathrm{~d}, J=2.0 \mathrm{~Hz}, 1 \mathrm{H}), 5.90-5.94$ $(\mathrm{m}, 4 \mathrm{H}), 4.38(\mathrm{dd}, J=8.5,12.0 \mathrm{~Hz}, 1 \mathrm{H}), 4.00(\mathrm{dd}, J=4.5,12.0 \mathrm{~Hz}$, $1 \mathrm{H}), 3.91(\mathrm{~s}, 3 \mathrm{H}), 3.90(\mathrm{~s}, 3 \mathrm{H}), 3.80$ (d, $J=15.5 \mathrm{~Hz}, 1 \mathrm{H}), 3.72-3.75$ (m, 1H), 3.59-3.63 (m, 1H), 3.36-3.39 (m, 1H), 3.16-3.18 (m, $1 \mathrm{H}), 2.95-2.98(\mathrm{~m}, 1 \mathrm{H})$, and $2.46-2.48(\mathrm{~m}, 1 \mathrm{H}) \mathrm{ppm} ;{ }^{13} \mathrm{C} \mathrm{NMR}$ $\left(125 \mathrm{MHz}, \mathrm{CDCl}_{3}\right) \delta_{\mathrm{C}} 178.6,149.5,149.4,146.5,144.0,143.7$, $143.6,136.8,136.4,134.3,131.8,121.3,114.4,111.3,107.9$, 101.5, $101.3(\times 2), 101.1,70.9,57.0,56.9,56.1,55.9,51.4,48.0$,
45.9, 41.6, and 35.9 ppm; HRMS-ESI calcd for $\mathrm{C}_{29} \mathrm{H}_{30} \mathrm{NO}_{8}[\mathrm{M}+$ $\mathrm{H}]^{+} 520.1971$, found 520.1952 .

(2S,3S)-6-(4"'-Aminobenzylamino)-2,6-dihydropeperomin E (16). Pale yellow oil (yield 72\%); 99.0\% HPLC purity; IR (thin film) $\nu_{\max } 3435.85,2924.67,2793.68,1770.11,1622.13,1508.11$, 1451.41, 1431.95, 1315.88, 1042.77, 928.64, and $814.32 \mathrm{~cm}^{-1} ;{ }^{1} \mathrm{H}$ NMR (500 MHz, $\left.\mathrm{CDCl}_{3}\right) 7.35(\mathrm{~d}, J=8.5 \mathrm{~Hz}, 2 \mathrm{H}), 7.29$ (d, $J=$ $8.5 \mathrm{~Hz}, 2 \mathrm{H}), 6.44$ (d, $J=2.0 \mathrm{~Hz}, 1 \mathrm{H}), 6.41(\mathrm{~d}, J=2.0 \mathrm{~Hz}, 2 \mathrm{H}), 6.38$ $(\mathrm{d}, J=2.0 \mathrm{~Hz}, 1 \mathrm{H}), 5.86-5.93(\mathrm{~m}, 4 \mathrm{H}), 4.29(\mathrm{dd}, J=8.5,12.0 \mathrm{~Hz}$, $1 \mathrm{H}), 4.07$ (dd, $J=4.5,12.0 \mathrm{~Hz}, 1 \mathrm{H}), 3.89(\mathrm{~s}, 3 \mathrm{H}), 3.83(\mathrm{~s}, 3 \mathrm{H}), 3.59$ (d, $J=15.5 \mathrm{~Hz}, 1 \mathrm{H}), 3.40-3.51(\mathrm{~m}, 2 \mathrm{H}), 3.12-3.17(\mathrm{~m}, 1 \mathrm{H}), 2.64$ (dd, $J=5.7,15.5 \mathrm{~Hz}, 1 \mathrm{H}), 2.42-2.45(\mathrm{~m}, 1 \mathrm{H})$, and $2.31-2.34(\mathrm{~m}$, 1H) ppm; ${ }^{13} \mathrm{C}$ NMR $\left(125 \mathrm{MHz}, \mathrm{CDCl}_{3}\right) \delta_{\mathrm{C}} 178.8,149.4,149.2$, 143.6, 143.4, 138.9, 137.0, 136.7, $134.1(\times 2), 129.2$, 128.4, 127.4, 108.1 ( $\times 2), 101.7,101.5,101.4,101.2,70.6,62.9,56.9,56.8,55.6$, 54.7, 44.6, 42.6, 42.1 ppm; HRMS-ESI calcd for $\mathrm{C}_{29} \mathrm{H}_{30} \mathrm{~N}_{2} \mathrm{O}_{8}[\mathrm{M}]^{+}$ 534.2002 , found 534.2092 .

(2S,3S)-6-(4'"'-Hydroxy-5'"'-methoxyphenethylamino)-2, 6-dihydropeperomin E (17). Pale yellow oil (yield 80\%); 98.4\% HPLC purity; IR (thin film) $\nu_{\max } 3441.18,2919.51,1762.85,1633.20,1514.00$, 1450.68, 1431.22, 1315.85, 1131.42, 1091.13, 1041.34, 926.12, and $819.81 \mathrm{~cm}^{-1}$; ${ }^{1} \mathrm{H}$ NMR $\left(500 \mathrm{MHz}, \mathrm{CDCl}_{3}\right) 6.85(\mathrm{~d}, J=8.5 \mathrm{~Hz}$, $1 \mathrm{H}), 6.71(\mathrm{~d}, J=2.0 \mathrm{~Hz}, 1 \mathrm{H}), 6.69(\mathrm{dd}, J=2.0,8.5 \mathrm{~Hz}, 1 \mathrm{H}), 6.47(\mathrm{~d}$, $J=2.0 \mathrm{~Hz}, 2 \mathrm{H}), 6.46(\mathrm{~d}, J=2.0 \mathrm{~Hz}, 1 \mathrm{H}), 6.43(\mathrm{~d}, J=2.0 \mathrm{~Hz}, 1 \mathrm{H})$, 5.92-5.96 (m, 4H), 4.30 (dd, $J=10.0,12.0 \mathrm{~Hz}, 1 \mathrm{H}), 3.92(\mathrm{~s}, 3 \mathrm{H})$, $3.90(\mathrm{~s}, 3 \mathrm{H}), 3.89$ (s, 3H), $3.87(\mathrm{dd}, J=4.5,12.0 \mathrm{~Hz}, 1 \mathrm{H}), 3.61(\mathrm{~d}, J$ $=14.5 \mathrm{~Hz}, 1 \mathrm{H}), 3.24-3.27(\mathrm{~m}, 1 \mathrm{H}), 2.80(\mathrm{dd}, J=10.0,15.5 \mathrm{~Hz}$, $1 \mathrm{H}), 2.72-2.76(\mathrm{~m}, 1 \mathrm{H}), 2.64-2.67(\mathrm{~m}, 2 \mathrm{H}), 2.48-2.51(\mathrm{~m}, 2 \mathrm{H})$, and 2.29 (dd, $J=7.0,15.5 \mathrm{~Hz}, 1 \mathrm{H}) \mathrm{ppm} ;{ }^{13} \mathrm{C} \mathrm{NMR}\left(125 \mathrm{MHz}, \mathrm{CDCl}_{3}\right.$ ) $\delta_{\mathrm{C}} 178.7,154.2,149.4(\times 2), 143.6(\times 2), 136.7,136.4,134.3,131.9$, $130.9,129.8(\times 2), 115.5(\times 2), 108.1,107.9,101.5,101.3,101.2$ $(\times 2), 70.9,57.1(\times 2), 56.9,56.1,51.2,48.0,45.9,41.5,35.3$ ppm; HRMS-ESI calcd for $\mathrm{C}_{31} \mathrm{H}_{34} \mathrm{NO}_{10}[\mathrm{M}+\mathrm{H}]^{+} 580.2183$, found 580.2151 .

(2S,3S)-6-(4"', $5^{\prime \prime \prime}$-Dihydroxyphenethylamino)-2,6-dihydropeperomin $E$ (18). Pale yellow oil (yield 75\%); 99.0\% HPLC purity; IR (thin film) $\nu_{\max } 3451.21,2916.40,1761.75,1632.11,1513.21,1448.38,1429.12$, 1305.87, 1130.12, 1071.03, 1031.54, 925.31, and $816.51 \mathrm{~cm}^{-1} ;{ }^{1} \mathrm{H}$ NMR $\left(500 \mathrm{MHz}, \mathrm{CDCl}_{3}\right) 6.85(\mathrm{~d}, J=2.0 \mathrm{~Hz}, 1 \mathrm{H}), 6.70(\mathrm{~d}, J=8.3 \mathrm{~Hz}$, $1 \mathrm{H}), 6.69(\mathrm{~d}, J=2.0 \mathrm{~Hz}, 1 \mathrm{H}), 6.68(\mathrm{dd}, J=2.0,8.3 \mathrm{~Hz}, 1 \mathrm{H}), 6.47(\mathrm{~d}, J$ $=2.0 \mathrm{~Hz}, 2 \mathrm{H}), 6.46(\mathrm{~d}, J=2.0 \mathrm{~Hz}, 1 \mathrm{H}), 6.43(\mathrm{~d}, J=2.0 \mathrm{~Hz}, 1 \mathrm{H}), 5.92-$ $5.96(\mathrm{~m}, 4 \mathrm{H}), 4.29$ (dd, $J=10.0,12.0 \mathrm{~Hz}, 1 \mathrm{H}), 3.90(\mathrm{~s}, 3 \mathrm{H}), 3.89(\mathrm{~s}$, $3 \mathrm{H}), 3.85(\mathrm{dd}, J=4.5,12.0 \mathrm{~Hz}, 1 \mathrm{H}), 3.60(\mathrm{~d}, J=15.0 \mathrm{~Hz}, 1 \mathrm{H}), 3.23-$ $3.27(\mathrm{~m}, 1 \mathrm{H}), 2.79$ (dd, $J=10.0,15.5 \mathrm{~Hz}, 1 \mathrm{H}), 2.71-2.74(\mathrm{~m}, 1 \mathrm{H})$, 2.60-2.65 (m, 3H), 2.49-2.52 (m, $1 \mathrm{H})$, and $2.30(\mathrm{dd}, J=7.0,15.5 \mathrm{~Hz}$, $1 \mathrm{H}) \mathrm{ppm} ;{ }^{13} \mathrm{C}$ NMR $\left(125 \mathrm{MHz}, \mathrm{CDCl}_{3}\right) \delta_{\mathrm{C}} 178.0,149.4,149.3,146.4$, 144.0, 143.6, 143.5, 136.8, 136.4, $134.2(\times 2), 131.7,121.2,114.3$ $(\times 2), 111.2,107.8,101.4,101.2,101.1(\times 2), 70.8,56.9,56.8,55.9$, 51.3, 48.0, 45.9, 41.6, 35.8 ppm; HRMS-ESI calcd for $\mathrm{C}_{30} \mathrm{H}_{32} \mathrm{NO}_{10}[\mathrm{M}$ $+\mathrm{H}]^{+}$566.2026, found 566.2024.

(2S,3S)-6-(4"'-Hydroxyphenethylamino)-2, 6-dihydropeperomin $E$ (19). Pale yellow oil (yield 75\%); 98.5\% HPLC purity; IR (thin film) $\nu_{\max } 3420.78,2926.96,1762.35,1633.09$, 1513.83, 1450.58, 1432.88, 1316.05, 1132.70, 1091.71, 1042.23, 926.38, and $827.66 \mathrm{~cm}^{-1}$; ${ }^{1} \mathrm{H}$ NMR (500 MHz, $\left.\mathrm{CDCl}_{3}\right) 7.03(\mathrm{~s}, 1 \mathrm{H}), 6.79(\mathrm{~s}$, $1 \mathrm{H}), 6.77(\mathrm{~s}, 1 \mathrm{H}), 6.74(\mathrm{~s}, 1 \mathrm{H}), 6.45(\mathrm{~d}, J=1.5 \mathrm{~Hz}, 1 \mathrm{H}), 6.41(\mathrm{~d}, J=$ $2.0 \mathrm{~Hz}, 2 \mathrm{H}), 6.39$ (d, J = 2.0 Hz, 1H), 5.92-5.97 (m, 4H), 4.28 (dd, 
$J=10.0,12.0 \mathrm{~Hz}, 1 \mathrm{H}), 3.92(\mathrm{~s}, 3 \mathrm{H}), 3.89$ (s, 3H), $3.83(\mathrm{dd}, J=8.5$, $12.0 \mathrm{~Hz}, 1 \mathrm{H}), 3.58$ (d, $J=14.5 \mathrm{~Hz}, 1 \mathrm{H}), 3.18-3.23$ (m, 1H), 2.73$2.80(\mathrm{~m}, 2 \mathrm{H}), 2.52-2.60(\mathrm{~m}, 3 \mathrm{H}), 2.45-2.48(\mathrm{~m}, 1 \mathrm{H})$, and $2.26(\mathrm{dd}$, $J=7.0,15.5 \mathrm{~Hz}, 1 \mathrm{H}) \mathrm{ppm} ;{ }^{13} \mathrm{C} \mathrm{NMR}\left(125 \mathrm{MHz}, \mathrm{CDCl}_{3}\right) \delta_{\mathrm{C}} 178.4$, 150.2 , 149.6, 149.4, 149.3, 143.7, 143.5, 138.5, 136.6, 136.1, 136.0, 134.4, 134.3, 134.2, 124.1 (×2), 108.0, 107.8, 101.6, 101.5, $101.1(\times 2), 71.0,57.0(\times 2), 56.2,50.2,48.0,45.9$, and $42.0 \mathrm{ppm}$; HRMS-ESI calcd for $\mathrm{C}_{30} \mathrm{H}_{32} \mathrm{NO}_{9}[\mathrm{M}+\mathrm{H}]^{+} 550.2077$, found 550.2049 .

General phosphate adducts formation procedure. To a flask containing a chloroformic solution $(0.1 \mathrm{M})$ of above amino derivative was added 1 equivalent of phosphate acid. Almost immediately, a white-colored precipitate formed, and the reaction mixture was stirred for an additional $60 \mathrm{~min}$. The precipitated salt was filtered and dried in vacuo to furnish the phosphate salt.

Computational methods for determining the absolute configurations. Conformational analysis was initially performed using Confab ${ }^{21}$ at Merck Molecular Force Field 94 (MMFF94) for all configurations of the amino-analogues. Room-temperature equilibrium populations were calculated, according to the Boltzmann distribution law (eqn (1)). The conformers with Boltzmann populations of over $1 \%$ were chosen for ECD calculations.

$$
\frac{N_{i}}{N}=\frac{g_{i} \mathrm{e}^{-\frac{E_{i}}{k_{\mathrm{B}} T}}}{\sum g_{i} \mathrm{e}^{-\frac{E_{i}}{k_{\mathrm{B}} T}}}
$$

where $N_{i}$ is the number of conformer $i$ with energy $E_{i}$ and degeneracy $g_{i}$ at temperature $T$, and $k_{\mathrm{B}}$ is the Boltzmann constant.

The theoretical calculation was performed using Gaussian 09 (Revision D.01. Gaussian Inc. Wallingford CT, USA). First, the chosen conformer was optimized at 6-31g* using the HartreeFock method, and then optimized at B3LYP/6-311G** in methanol using the polarizable continuum model (PCM). The theoretical calculation of ECD was conducted in methanol using time-dependent density functional theory (TD-DFT) at the same theory level. Rotatory strengths for a total of 30 excited states were calculated. The ECD spectrum was simulated in SpecDis $^{22}$ by overlapping Gaussian functions for each transition, according to (eqn (2)):

$$
\Delta \varepsilon(E)=\frac{1}{2.297 \times 10^{-39}} \times \frac{1}{\sqrt{2 \pi \sigma}} \sum_{i}^{A} \Delta E_{i} R_{i} \mathrm{e}^{-\left(\frac{E-E_{i}}{2 \sigma}\right)^{2}}
$$

where $\sigma$ represents the width of the band at $1 / e$ height, and $\Delta E_{i}$ and $R_{i}$ are the excitation energies and rotatory strengths for transition $i$, respectively.

The parameters of $\sigma$ and UV-shift for each configuration are listed in Table S3.†

\section{Biological assay}

Cell culture. HL-60, KG-1, and THP-1 cells were purchased from KeyGen Biotechnology (Nanjing, Jiangsu, China). KG-1a and hBMSCs were purchased from BeNa Culture Collection Biotechnology (Suzhou, Jiangsu, China). All cells were grown in complete medium, consisting of 90\% RPMI 1640 medium (Hyclone, GE, USA) and 10\% foetal bovine serum (Gibco, Invitrogen, USA), supplemented with $100 \mathrm{U} \mathrm{mL}^{-1}$ penicillin and 100 $\mu \mathrm{g} \mathrm{mL} \mathrm{m}^{-1}$ streptomycin in $25 \mathrm{~cm}^{2}$ flasks (Corning, New York, USA) at $37{ }^{\circ} \mathrm{C}$ in a $5 \% \mathrm{CO}_{2}$ incubator (Thermo Scientific, Massachusetts, USA).

Differentiation assay. KG-1a cells were collected and washed with PBS twice and then diluted with PBS $\left(1 \times 10^{7} / 0.1 \mathrm{~mL}\right)$. After adding CD38-FITC monoclonal antibody (Miltenyi Biotech, Germany), the cells were incubated at $37{ }^{\circ} \mathrm{C}$ for $30 \mathrm{~min}$ in the dark and washed with prechilled PBS solution. Then, the cells were resuspended and enriched by magnetic-activated cell sorting (MACS), using Midi MACS starting kits and anti-FITC microbeads (Miltenyi Biotec), according to the manufacturer's protocol. After the enrichment, the purity of the $\mathrm{CD} 34^{+} \mathrm{CD} 38^{-}$ cells was assessed with the BD Accuri ${ }^{\text {TM }}$ C6 flow cytometer (BD Biosciences, New Jersey, USA), after staining with anti-CD38FITC and anti-CD34-phycoerythrin (PE) monoclonal antibodies (Miltenyi Biotech), according to the manufacturer's protocol.

Cell growth inhibition assay. HL-60, KG-1, THP-1, and KG-1a CD $34^{+} \mathrm{CD}^{-} 8^{-}$cells were seeded at $2 \times 10^{3}$ cells per well in 96well plates (Corning, New York, USA) and incubated for $2 \mathrm{~h}$. Then, the cells were treated with different concentrations of PepE, its amino-derivatives, or the positive control, PTL, at $37^{\circ} \mathrm{C}$ in the $5 \% \mathrm{CO}_{2}$ incubator for $48 \mathrm{~h}$. After $48 \mathrm{~h}, 10 \mu \mathrm{L}$ of Cell Counting Kit-8 solution (CCK-8, Yifeixue Biotech Ltd., Nanjing, China) was added to each well and incubated at $37{ }^{\circ} \mathrm{C}$ for $2 \mathrm{~h}$. The absorbance at $450 \mathrm{~nm}$ was finally measured with a microplate reader (Thermo Scientific, Massachusetts, USA).

Apoptosis assay. Cell apoptosis was assessed using an annexin V-FITC/PI (propidium iodide) double-staining cell apoptosis detection kit (KeyGen Tech., Nanjing, China). The enriched $\mathrm{KG}-1 \mathrm{a} \mathrm{CD} 34^{+} \mathrm{CD} 38^{-}$cells were seeded into 6-well tissue culture dishes at $1 \times 10^{6}$ cells per well and treated with PepE or its derivative 6 at 1 and $5 \mu \mathrm{M}$. After incubation at $37{ }^{\circ} \mathrm{C}$ for $48 \mathrm{~h}$, the cells were collected and diluted to $10^{6}$ cells per $\mathrm{mL}$ well with $1 \times$ binding buffer. Five microliters of annexin V-FITC and $5 \mu \mathrm{L}$ PI reagents were added to the drug-treated cells and allowed to stain in the dark for $15 \mathrm{~min}$ for the determination of early and late apoptosis. The cells were detected according to the manufacturer's instructions using the BD Accuri ${ }^{\text {тм }}$ C6 flow cytometer (BD Biosciences).

\section{Results and discussion}

\section{Chemistry}

In this manuscript, we report the synthesis of a series of PepE analogues derived from open chain secondary aliphatic (2-7), cyclic secondary aliphatic (8-12), and several open chain or arylalkyl primary (13-19) amines. The choice of amines was based on the results recently reported in studies on PTL derivatives. ${ }^{16,18}$ The synthesis of PepE derivatives 2-19 was carried out using the procedures outlined in Scheme 1.

PepE was obtained from the $\mathrm{CH}_{2} \mathrm{Cl}_{2}$ extract of whole $P$. dindygulensis plants, according to methodology described previously. $^{8}$ Its structure was unambiguously confirmed by 


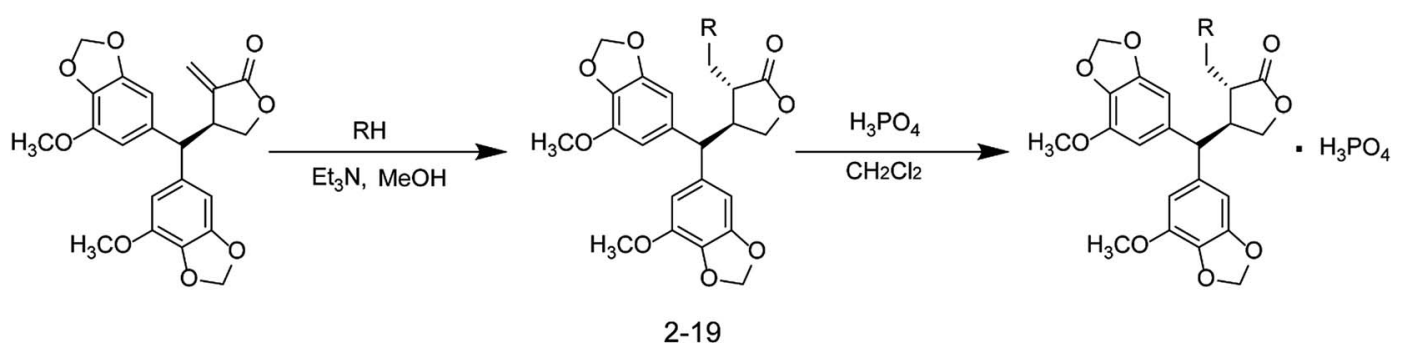

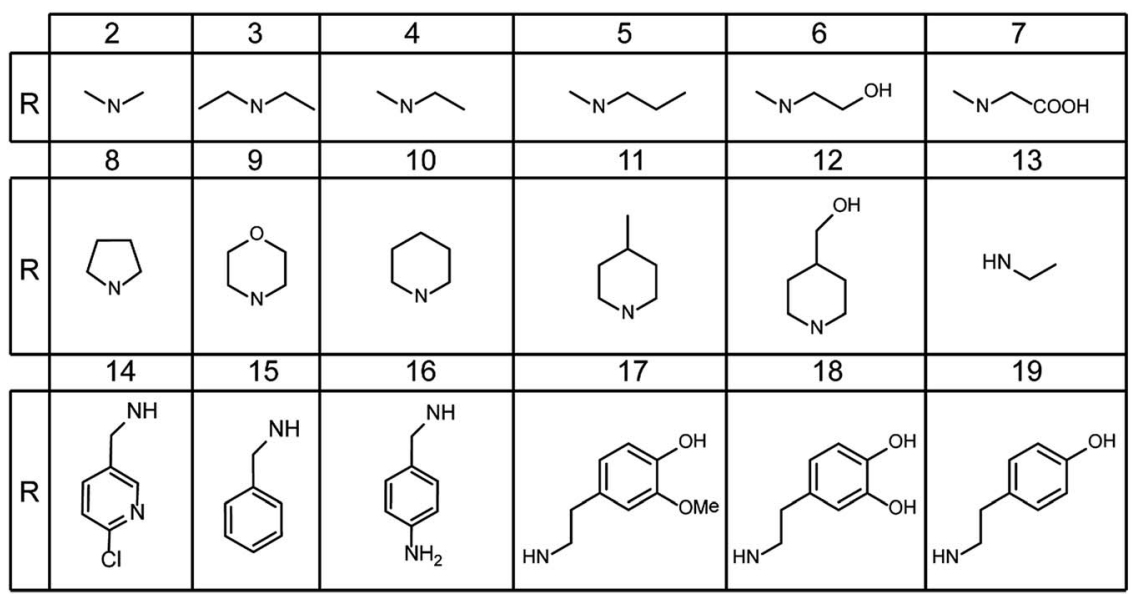

Scheme 1 Synthesis of 18 peperomin E amino-analogs.

a combination of spectroscopic methods, including infrared spectroscopy, NMR, and high-resolution mass spectrometry.

To obtain a library of water-soluble PepE analogues, $0.2 \mathrm{mM}$ of PepE in methanol was treated with one equivalent of an appropriate primary or secondary amine, using trimethylamine as a catalyst at room temperature $\left(15-20^{\circ} \mathrm{C}\right)$, which led to a significant consumption of PepE within $8 \mathrm{~h}$. The reaction product was purified by flash column chromatography. A good yield (65-92\%) of product was obtained, but unreacted PepE persisted even after prolonged reaction time, addition of excess amine, or increased temperature.

The amino PepE analogues thus obtained (2-19) were then converted into water-soluble salts, thereby increasing their water solubility. Multiple inorganic/organic acid were tested for salt formation including fumarate acid, citric acid, sulphate acid, phosphate acid and hydrochloride acid. The phosphate salts have the best crystallinity, and relatively low hygroscopic properties, therefore selected it as the salt-forming acid.

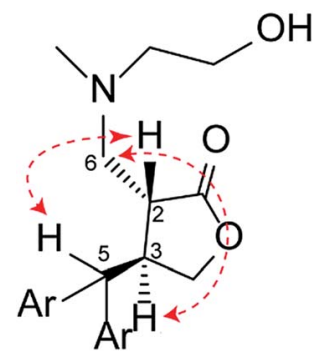

Fig. 2 The key NOE correlations of compound 6.
The ${ }^{1} \mathrm{H}$ NMR spectra of the synthesised analogues showed the absence of the two olefinic proton resonances at 6.15 and $4.94 \mathrm{ppm}$ in the PepE molecule. ${ }^{6-8}$ The appearance of new methine and methylene resonances in the distortionless enhancement by polarization transfer (DEPT) spectrum of ${ }^{13} \mathrm{C}$ NMR was also apparent, indicating that amination at C-6 of the exocyclic double bond had occurred by Michael-addition reactions. The newly formed C-2 chiral carbon was found to be highly stereospecific based on the nuclear Overhauser effect spectroscopy (NOESY) spectrum. The cross-peaks of $\mathrm{H}-6 / \mathrm{H}-3$ and $\mathrm{H}-2 / \mathrm{H}-5$, as well as the absence of NOESY correlation between $\mathrm{H}-6$ and $\mathrm{H}-5$ (Fig. 2), indicated a trans configuration at the $\gamma$-butyrolactone ring of the synthesised products. Thus, there were only two possible absolute configurations of the $\gamma$ butyrolactone ring (Fig. 3), namely $(2 S, 3 S)$ or $(2 R, 3 R)$. The structures of the synthesised products were elucidated by NMR, ultraviolet spectroscopy, and mass spectrometry data; all NMR

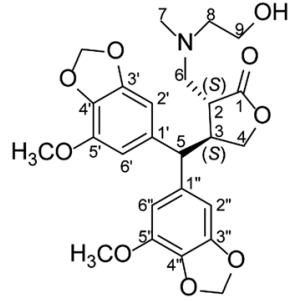

(a)

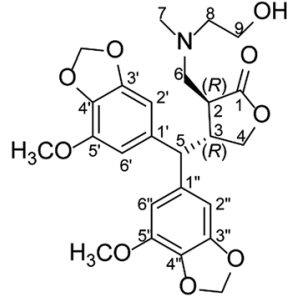

(b)
Fig. 3 Two possible absolute configurations of compound 6, namely $(2 S, 3 S)-6(a)$ or $(2 R, 3 R)-6(b)$. 
signals were accordingly assigned based on heteronuclear multiple bond correlation (HMBC) and heteronuclear singlequantum correlation (HSQC) experiments (see Experimental sections).

Our repeated attempts to obtain suitable crystals for X-ray diffraction were unsuccessful, and so the absolute configurations of the $\gamma$-butyrolactone ring of the analogues were established by comparing the experimental and theoretical electronic circular dichroism (ECD) spectra predicted using the time-dependent density functional theory (TDDFT) at the B3LYP/6-311G** level. Take compound 6 for instance, the energies and populations of all dominative conformers of 6 are provided in Table S1. $\dagger$ The energies of the all conformers of 6 optimized at B3LYP/6-311G** in methanol are provided in Fig. 4 and Table S2. $\uparrow$ The ECD spectrum of 6 showed intense positive Cotton effects at 220 and $280 \mathrm{~nm}$, as well as weakly negative Cotton effects at $254 \mathrm{~nm}$, which were similar to those of reported peperomin B. ${ }^{22,23}$ This indicated that 6 might share the same configuration as peperomin $\mathrm{B}$, which is $(2 S, 3 S) .{ }^{24}$ Moreover, the overall pattern of the simulated ECD spectrum for the $(2 S, 3 S)$ stereoisomer was in good agreement with the measured spectrum of 6 (Fig. 5, the parameters of $\sigma$ and UVshift for each configuration are listed in Table S3†). Therefore, the absolute configuration of $\mathbf{6}$ was unambiguously assigned as $2 S, 3 S$. In addition, we have confirmed the $(2 S, 3 S)$ configuration in many of our synthesised products by comparing the experimental and calculated ECD spectra.

The exclusive formation of the $2 S$ configuration in the $\gamma$ butyrolactone ring, according to the literature, is due to the protonation of the enolate formed during Michael addition, which occurs exclusively from the exo face of the $\gamma$-butyrolactone ring, resulting in an $S$ configuration at C-2 in the product. ${ }^{16}$

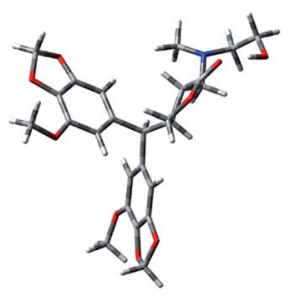

Conformer 1 B3IYP/6-311G** $0.0 \mathrm{kcal} / \mathrm{mol}(96.57 \%)$

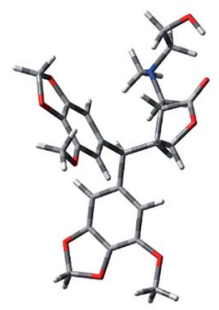

Conformer 3 B3IYP/6-311G** $+4.61 \mathrm{kcal} / \mathrm{mol}(0.04 \%)$

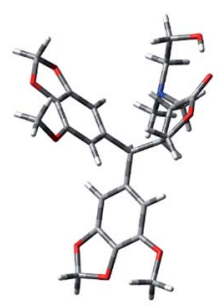

Conformer 2 B3IYP/6-311G** $+2.53 \mathrm{kcal} / \mathrm{mol}(1.34 \%)$

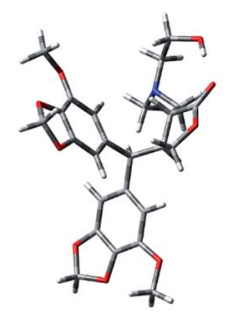

Conformer 4 B3IYP/6-311G** $+2.28 \mathrm{kcal} / \mathrm{mol}(2.05 \%)$
Fig. 4 Optimized structures and relative energies of the four lowestenergy conformers of $(2 S, 3 S)-6$ at the B3LYP/6-311G** level in methanol.

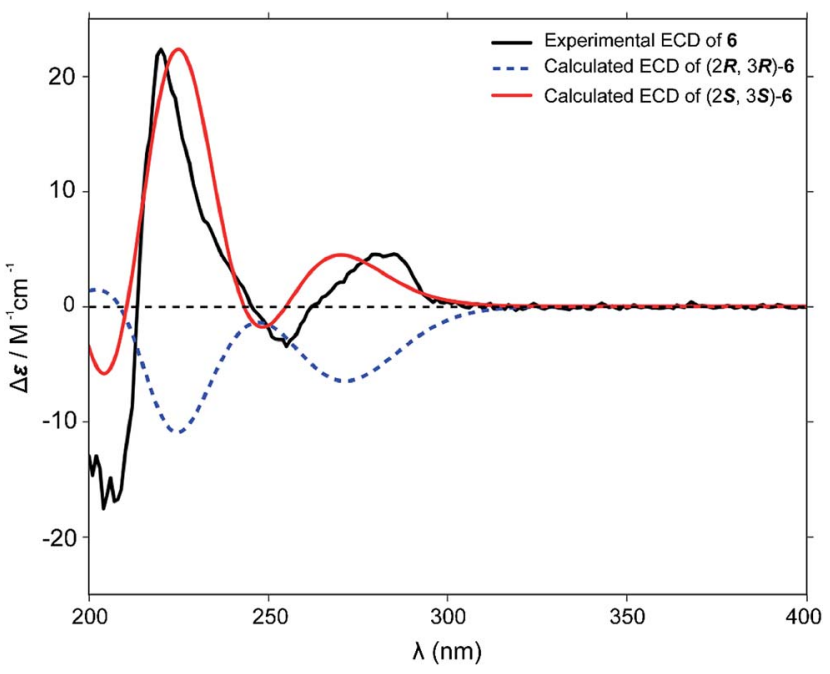

Fig. 5 Comparison of the ECD spectrum of measured compound 6 (black line) with the calculated [B3LYP/6-311G**] ECD spectra of the Boltzmann average of the lowest conformers of the corresponding $(2 R, 3 R)-6$ (blue dot line) and $(2 S, 3 S)-6$ (red line).

\section{Biological activity}

To evaluate the anti-AML activity of PepE and its amino analogues, all of the synthesised compounds were tested against cultured cell lines, including KG-1 (acute myelogenous leukaemia), HL-60 (acute promyelocytic leukaemia), THP-1 (acute monocytic leukaemia), and KG-1a (acute myelogenous leukaemia), using PTL (a drug that selectively ablates acute leukaemia stem cells and has advanced to a phase I clinical trial for treatment of $\mathrm{AML}^{25}$ ) as a positive control. hBMSCs were used as a model to test the toxicity of PepE and its derivatives against normal cells. It has been reported that bearing a $\mathrm{CD} 34^{+} \mathrm{CD} 38^{-}$immunophenotype is a characteristic of LSCs. $^{26}$ From our results of flow cytometry, the percentage of $\mathrm{CD}_{3} 4^{+}$cells in the KG-1a cell line was $98.9 \%$, and the percentage of $\mathrm{CD} 34^{+} \mathrm{CD} 38^{-}$was $50.1 \%$. These results corroborated those reported by Liu et al. ${ }^{27}$ confirming that KG-1a represents an AML stem cell-like line. Then, $\mathrm{CD} 34^{+} \mathrm{CD} 38^{-}$cells were enriched from the KG-1a cell line using MACS, as reported previously by Zhang et al. ${ }^{28}$ The percentage of the $\mathrm{CD} 34^{+} \mathrm{CD} 38^{-}$cell population in the KG-1a cells was significantly increased after sorting (92.1 \pm $1.3 \%$ versus $50.1 \pm 5.5 \%$ before sorting).

Eighteen amino analogues were then studied with a CCK-8 cytotoxicity assay against the KG-1, HL-60, THP-1, and KG-1a $\mathrm{CD} 4^{+} \mathrm{CD} 38^{-}$cell lines. Preliminary cytotoxic screening of the analogues was performed at $10 \mu \mathrm{M}$. The analogues that exhibited greater than $50 \%$ growth inhibition at $10 \mu \mathrm{M}$ were further assayed at varying concentrations to generate the $\mathrm{IC}_{50}$ values shown in Table 1. It is evident from Table 1 that the parent molecule, PepE, exhibited similar cytotoxicity against AML and LSC cells as the positive control, PTL, did, while its toxicity at $50 \mu \mathrm{M}$ against hBMSCs was half that of PTL. It is also evident from Table 1 that several of the amino-PepE products exhibited increased cytotoxicity toward AML and LSC cells compared to that of the parent molecule, while others showed decreased activity. The structure and activity relationship (SAR) of these synthetic derivatives is discussed below. 
Table 1 Antileukemic activities of PepE and its amino derivatives

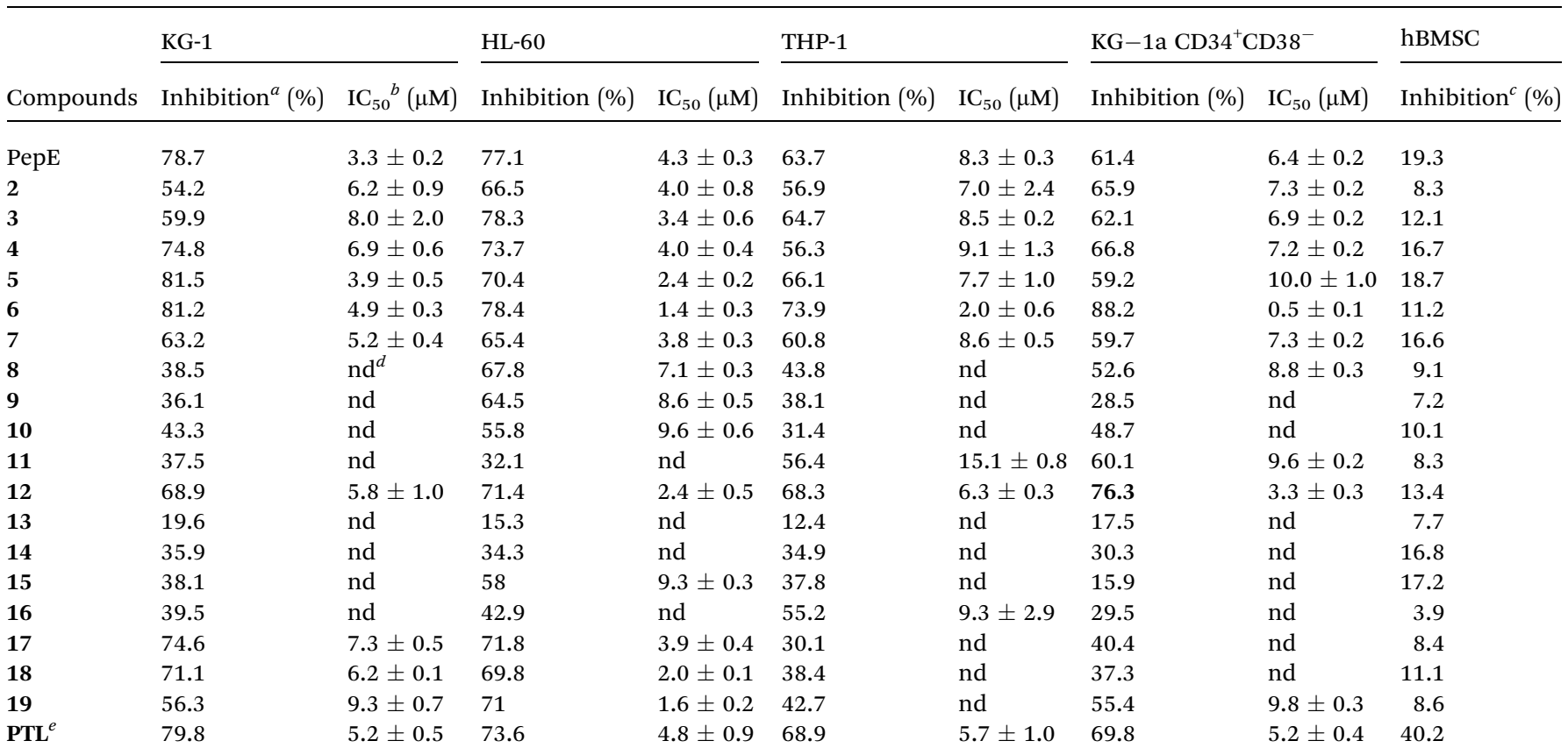

${ }^{a} \%$ Inhibition refers to growth inhibition measured at $10 \mu \mathrm{M}$ concentration. ${ }^{b}$ All values are the mean of three independent experiments. ${ }^{c} \%$ Inhibition refers to growth inhibition measured at $50 \mu \mathrm{M}$ concentration. ${ }^{d}$ nd, not determined in the given concentration range. ${ }^{e}$ PTL, parthenolide, used as a positive control.

The open-chain secondary aliphatic amine series $(2-5,7)$ was found to be nearly as active as was the parent molecule, PepE. However, compound 6, with a hydroxyl group replacing the 10methyl group of compound $\mathbf{5}$, was the exception. The cytotoxicity of compound $6\left(\mathrm{IC}_{50}=0.5 \mu \mathrm{M}\right)$ significantly improved up to 13-fold compared to that of the parent molecule against KG-1a
$\mathrm{CD} 34^{+} \mathrm{CD} 38^{-}$cells, while there was little effect on the viability of normal hBMSCs (inhibition rate of $11.2 \%$ at $50 \mu \mathrm{M}$ ).

The toxicity level against AML cells of the cyclic aliphatic amine series (8-11), in contrast, was significantly decreased when compared with that of the parent molecule. However, replacing the 3-methyl piperidine moiety in compound $\mathbf{1 1}$ with a 3-hydroxymethyl piperidine moiety to form compound $\mathbf{1 2}$

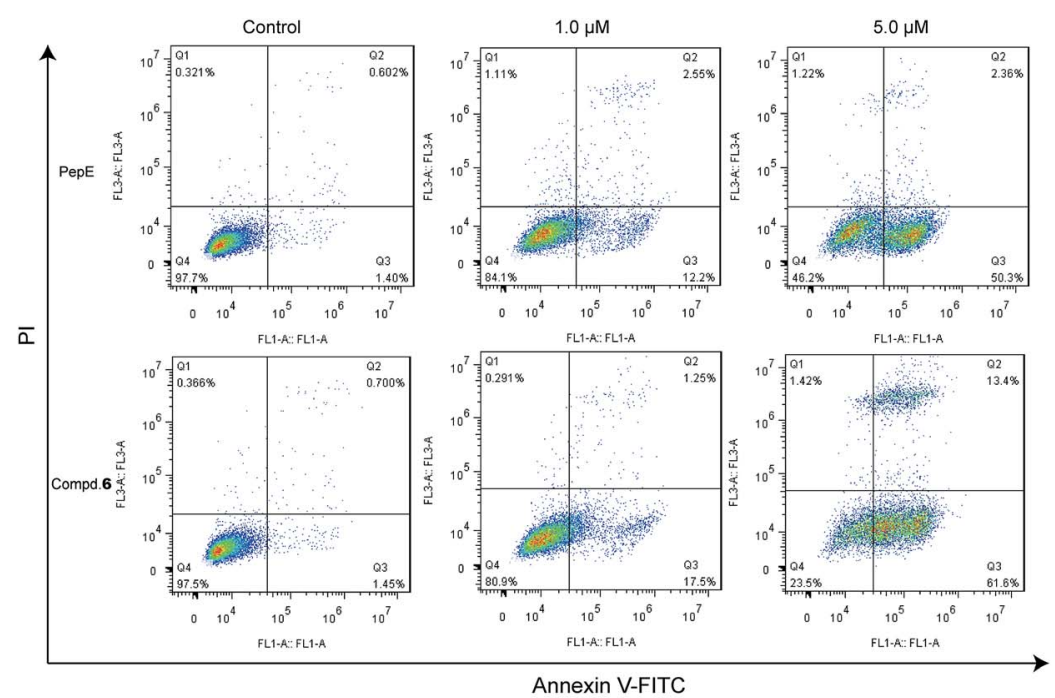

(a)

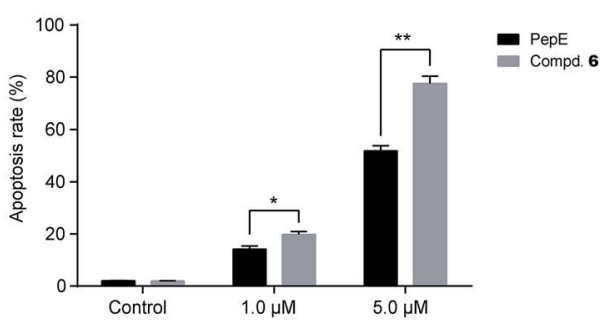

(b)

Fig. 6 Compound 6 and PepE induced apoptosis in KG-1a CD34 ${ }^{+} \mathrm{CD} 38^{-}$cells. (a) Apoptosis induced by compound 6 in KG-1a CD34 ${ }^{+} \mathrm{CD} 38^{-}$ cells at different concentration by flow cytometry analysis. After the treatment of 6 for $12 \mathrm{~h}$, the cells were stained with annexin $\mathrm{V}$-FITC and PI for 15 min for flow cytometry analysis. The $X$ axis represents annexin V-FITC signal intensity and the $Y$ axis represents PI signal intensity. (b) Data were presented as mean \pm SD. 
increased the cytotoxicity against HL-60 and KG-1a $\mathrm{CD} 34^{+} \mathrm{CD} 38^{-}$cells $\left(\mathrm{IC}_{50}=2.4\right.$ and $3.3 \mu \mathrm{M}$, respectively) up to 2 -fold compared to that of PepE and >3-fold compared to that of compound 11; the safety level of compound 12 was approximately the same as that of PepE $(13.4 \%$ at $50 \mu \mathrm{M})$.

The primary amine series (13-19) was generally less active against AML cells when compared with that of the parent molecule and the secondary amine series; some analogues (13, 14) displayed low growth inhibition at the preliminary screening concentration. However, introduction of tyramine (18) or its hydroxyl derivatives (19) into the PepE structure resulted in a significant increase in cytotoxicity against HL-60 cells $\left(\mathrm{IC}_{50}=2.0\right.$ and $1.6 \mu \mathrm{M}$, respectively), increasing cytotoxicity up to 2-fold compared to that of the parent molecule.

In summary, 18 amino-PepE products were synthesised and evaluated for anti-leukaemic activity against AML cell lines in culture. The phosphate salts of all the synthesised compounds were more water soluble than PepE was, especially compound 6, which exhibited 1000-fold greater water-solubility than did PepE. A range of anti-leukaemic activities was observed, with several products exhibiting enhanced activities compared to that of PepE.

Compound $\mathbf{6}$ was the most potent compound of all synthesised amino analogues, especially against the immature AML stem cells, KG-1a CD34 ${ }^{+} \mathrm{CD} 38^{-}$, inducing $80-90 \%$ cell death at $10 \mu \mathrm{M}$, and affording an $\mathrm{IC}_{50}$ value of $0.5 \mu \mathrm{M}$ with little effect on hBMSCs. Therefore, the mechanism of action of compound 6 was further explored in preliminary experiments. Apoptosis was analysed by flow cytometric assay. The percentages of apoptotic cells were $18.7 \%$ and $75.1 \%$ in the KG-1a $\mathrm{CD}^{+} 4^{+} \mathrm{CD} 38^{-}$cells after treatment with $1 \mu \mathrm{M}$ and $5 \mu \mathrm{M}$, respectively, and were significantly higher than those of the parent molecule (Fig. 6). This result indicated that 6 can significantly induce apoptosis of leukaemia stem cells.

As reported by many clinicians, the $\mathrm{CD} 34^{+} \mathrm{CD} 38^{-} \mathrm{AML}$ cell subpopulation is frequently impervious to conventional chemotherapy, making it largely responsible for relapse of AML. Therefore, targeting and eliminating these cells has been proposed as a promising strategy for treatment of AML to decrease the rate of relapse or drug-resistance. ${ }^{2}$ However, small molecules that can selectively target LSCs are rare and still at early stages of development. As reported in this manuscript, compound 6 may be considered as a new lead compound to eliminate these AML stem cells. Further pharmacological and pharmacokinetic evaluation of this compound is underway.

\section{Conclusions}

In conclusion, for the first time, we have reported a newly found naturally occurring agent, PepE, which targets leukaemia stem cells. A series of 18 novel amino-derivatives of PepE was then synthesised and evaluated for anti-AML and anti-LSC activity. All of the compounds showed higher water solubility than the parent molecule. More importantly, this study found that one of the amino derivatives, compound $\mathbf{6}$, was more potent than was the parent drug, PepE, against the LSC-like cells (KG-1a $\mathrm{CD}_{4}{ }^{+} \mathrm{CD} 38^{-}$), with no apparent toxicity on hBMSCs.
According to the above results, the following SAR of the PepE amino derivatives can be formulated: (1) the secondary openchain aliphatic amino analogues generally exhibited better activity than the cyclic aliphatic and primary amino analogues did; and (2) introducing a hydroxyl group at the methyl group of the secondary open-chain amine adduct could significantly potentiate the activity against AML stem cells, and result in the most active compound 6 .

Based on the above results, further optimization and biological evaluation of $\mathbf{6}$ as a promising agent targeting LSC are ongoing in our laboratory, and the results will be reported shortly.

\section{Conflicts of interest}

The authors declare no conflict of interest.

\section{Acknowledgements}

This work was supported by Grants from the Natural Science Foundation of China (No. 81402812), the Natural Science Foundation of Jiangsu Province (No. BK20130954) and the Priority Academic Program Development of Jiangsu Higher Education Institutions.

\section{References}

1 H. Dohner, D. J. Weisdorf and C. D. Bloomfield, N. Engl. J. Med., 2015, 373, 1136-1152.

2 U. Testa, Ann. Hematol., 2011, 90, 245-271.

3 A. K. Burnett, N. Russell, R. K. Hills, N. Panoskaltsis, A. Khwaja, C. Hemmaway, P. Cahalin, R. E. Clark and D. Milligan, Leukemia, 2015, 29, 1312-1319.

4 D. C. Hassane, M. L. Guzmann, C. Corbert, X. J. Li, R. Abboud, F. Young, J. L. Liesveld, M. Carroll and C. T. Jordan, Blood, 2008, 111, 5654-5662.

5 T. R. Govindachari, G. N. Krishna Kumari and P. D. Partho, Phytochemistry, 1998, 49, 2129-2131.

6 M. G. Lloyd, R. J. K. Taylor and W. P. Unsworth, Org. Biomol. Chem., 2016, 14, 8971.

7 X. Z. Wang, Y. Cheng, K. L. Wang, R. Liu, X. L. Yang, H. M. Wen, C. Chai, J. Y. Liang and H. Wu, Cancer Sci., 2016, 10, 1506-1519.

8 X. Z. Wang, Y. Cheng, H. Wu, N. Li, R. Liu, X. L. Yang, Y. Y. Qiu, H. M. Wen and J. Y. Liang, Phytomedicine, 2016, 23, 818-827.

9 A. Ghantous, H. Gali-Muhtasib, H. Vuorela, N. A. Saliba and N. Darwiche, Drug Discovery Today, 2010, 15, 668-678.

10 X. Z. Wang, H. M. Wen, C. Chai, W. Y. Zhang, M. Gao, R. Liu, H. Wu and J. Y. Liang, Biomed. Chromatogr., 2017, 31, e3930.

11 J. R. Woods, H. Mo, A. A. Bieberich, T. Alavanja and D. A. Colby, MedChemComm, 2013, 4, 27-33.

12 A. Kumar, D. Kumar, A. K. Maurya, Y. S. Padwad and V. K. Agnihotri, Phytochem. Lett., 2016, 18, 117-121.

13 S. Zimmermann, G. Franche, M. D. Mieri, Y. Yoshimoto, T. Usuki, R. Nthambeleni, C. J. Parkinson, C. van der Westhuyzen, M. Kaiser, M. Hamburger and M. Adams, Molecules, 2014, 19, 3523-3538. 
14 X. J. Wang, X. M. Zhang, B. B. Zheng, N. Hu, W. D. Xie and K. Row, Nat. Prod. Res., 2015, 29, 756-763.

15 Y. H. Ding, H. E. Gao, Y. Zhang, Y. Li, N. Vasdev, Y. D. Gao, Y. Chen and Q. Zhang, J. Hematol. Oncol., 2016, 9, 93-99.

16 S. Nasim and P. A. Crooks, Bioorg. Med. Chem. Lett., 2008, 18, 3870-3873.

17 H. Nakshatri, H. N. Appaiah, M. Anjanappa, D. Gilley, H. Tanaka, S. Badve, P. A. Crooks, W. Mathews, C. Sweeney and P. Bhat-Nakshatri, Cell Death Dis., 2015, 6, e1608.

18 M. Moumou, A. El Bouakher, H. Allouchi, A. El Hakmaoui, A. Benharref, V. Mathieu, G. Guillaumet and M. Akssira, Bioorg. Med. Chem. Lett., 2014, 24, 4014-4018.

19 Z. Yang, B. Kuang, N. Kang, Y. Ding, W. Ge, L. Lian, Y. Gao, Y. Wei, Y. Chen and Q. Zhang, Eur. J. Med. Chem., 2017, 127, 296-304.

20 M. L. Guzman, R. M. Rossi, L. Karnischky, X. J. Li, D. R. Peterson, D. S. Howard and C. T. Jordan, Blood, 2005, 105, 4163-4168.

21 N. M. OBoyle, T. Vandermeersch, C. J. Flynn, A. R. Maguire and G. R. Hutchison, J. Cheminf., 2011, 3, 3-8.
22 T. Bruhn, A. Schaumlffl, Y. Hernberger and G. Bringmann, Chirality, 2013, 25, 243-249.

23 C. M. Chen, F. Y. Fan, M. T. Chen and T. J. Lee, Heterocycles, 1989, 29, 411-414.

24 T. J. Lee and T. Y. Lee, Acta Crystallogr., Sect. C: Cryst. Struct. Commun., 1990, 46, 338-340.

25 A. Ghantous, A. Sinjab, Z. Herceg and N. Darwiche, Drug discovery today, 2013, 18, 894-905.

26 M. Terwijn, W. Zeijlemaker, A. Kelder, A. P. Rutten, A. N. Snel, W. J. Scholten and G. J. Ossenkoppele, PLoS One, 2014, 9, e107587.

27 Y. Liu, F. Chen, S. Wang, X. Guo, P. Shi and W. Wang, Cell Death Dis., 2013, 4, e948.

28 Y. Zhang, S. Y. Zhou, H. Z. Yan, D. D. Xu, H. X. Chen, X. Y. Wang, X. Wang, Y. T. Liu, L. Zhang, S. Wang, P. J. Zhou, W. Y. Fu, B. B. Ruan, D. L. Ma, Y. Wang, Q. Y. Liu, Z. Ren, Z. Liu, R. Zhang and Y. F. Wang, Sci. Rep., 2016, 6, 19995. 